\title{
Adeno-associated and lentiviral vector production in 2D and 3D formats with adherent cells in chemically defined, blood-free media
}

\author{
Sofia Pezoa ${ }^{1}$, Randall Alfano ${ }^{2}$, Atherly Pennybaker ${ }^{3}$, Nathan Hazi ${ }^{4}$, and Andrew \\ Laskowski ${ }^{5}$ \\ ${ }^{1}$ InVitria \\ ${ }^{2}$ Ventria Bioscience \\ ${ }^{3}$ InVItria \\ ${ }^{4}$ Pall Corporation \\ ${ }^{5}$ Pall Corp
}

September 27, 2021

\begin{abstract}
Large scale manufacturing of viral vectors or vaccines with adherent cells still relies heavily on the inclusion of fetal bovine serum for the growth and production phases. The inclusion of serum presents numerous problems with the undefined chemical makeup, the undesirable safety profile, and the constraints and limitations on the global supply. Despite these challenges, alternatives to serum for adherent cells have been limited; however, advances in large-scale production of recombinant human proteins have enabled the advancement of blood-free media that can support adherent cell growth. In order to circumvent the need for serum in adherent platforms, we developed a serum and blood-free, chemically defined medium specific for adherent human epithelial kidney cells and evaluated growth kinetics as well as viral vector production with associated adenovirus and lentivirus. We observed doubling times equal to or faster than doubling times observed in serum containing medium. We also demonstrate transfection efficiencies and viral titers that are equivalent to or higher than that of serum. Our results demonstrate that fetal bovine serum is not required for culture of adherent HEK cells, and that a serum-free, blood-free, chemically defined approach can be reliably implemented in the production of viral vectors for gene therapy.
\end{abstract}

\section{Hosted file}

AAV_LVmanuscript_9-22-21.docx available at https://authorea.com/users/438008/articles/539322adeno-associated-and-lentiviral-vector-production-in-2d-and-3d-formats-with-adherentcells-in-chemically-defined-blood-free-media 

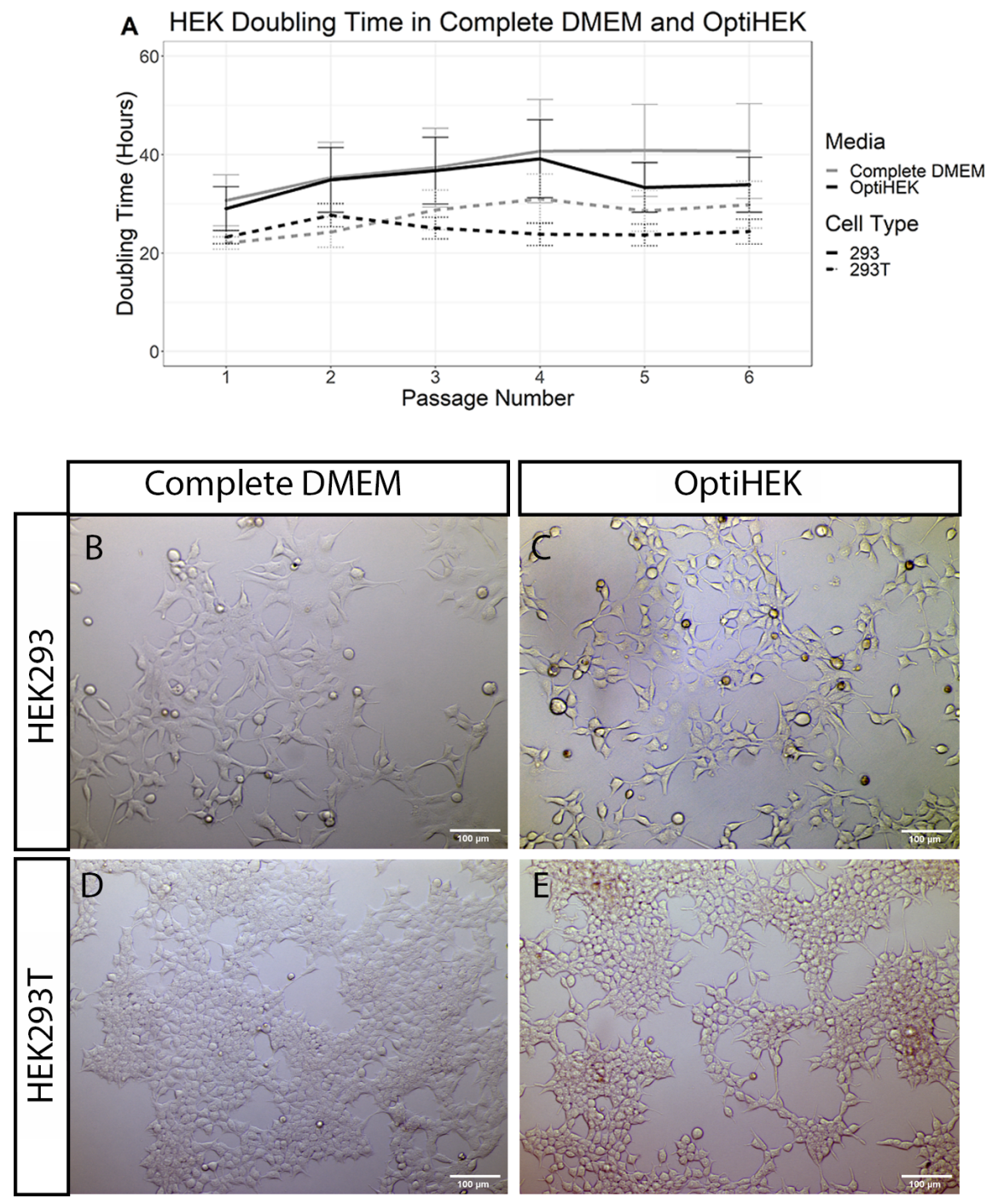

Transfection efficiency of HEK cells in complete DMEM and OptiHEK

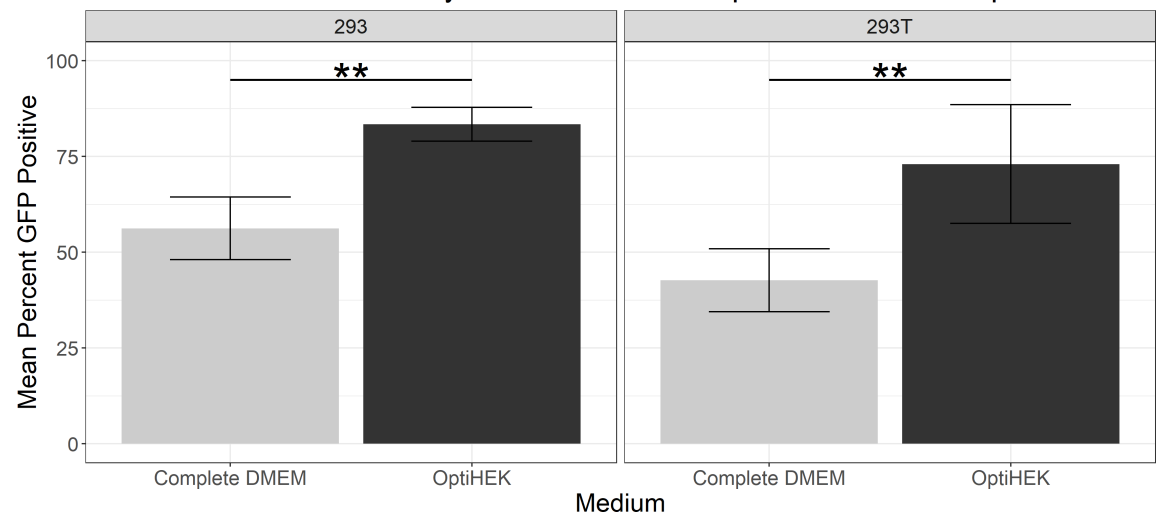


A Estimated AAV2 functional titer
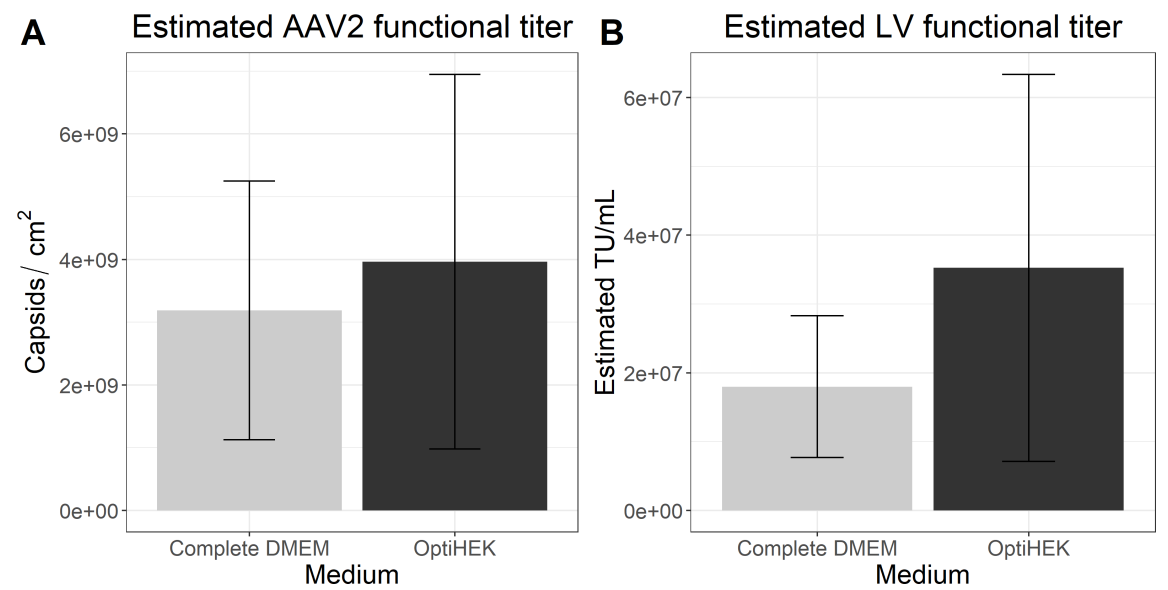

A Cell Density from Aber Biomass probe
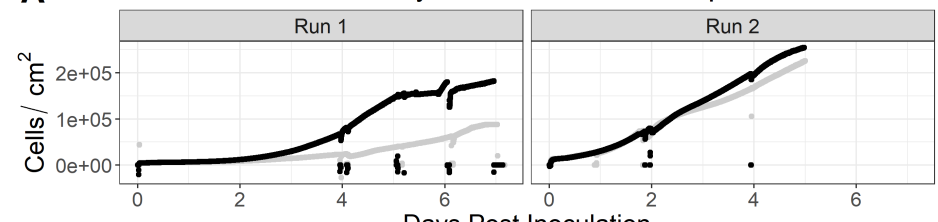

Production Media

- Complete DMEM

Days Post Inoculation

\section{B Glucose Consumption}

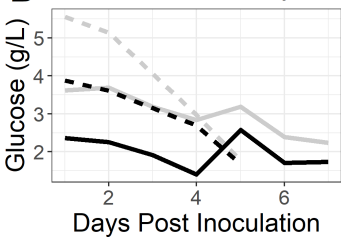

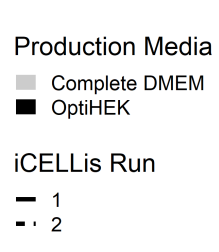

Production Media

- OptiHEK

iCELLis Run

-1
$-\cdot 2$

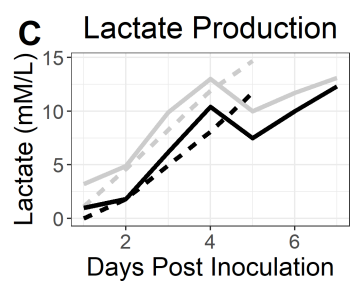

Production Media

Complete DMEM

- OptiHEK

iCELLis Run

$=1$

iCELLis Bioreactor LV Functional Titer in Complete DMEM and OptiHEK

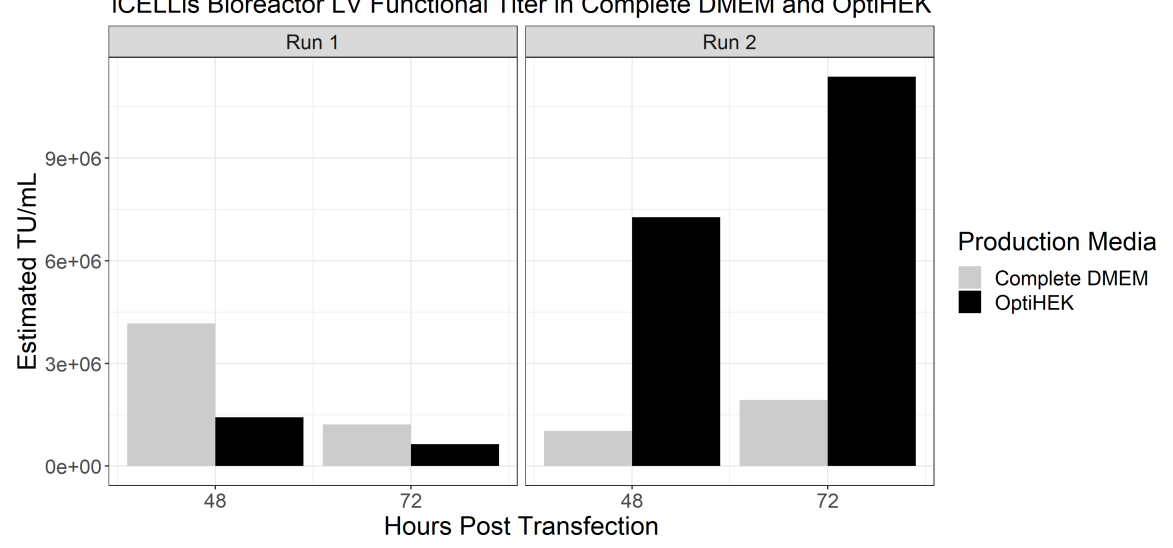

